\title{
Problem-Solving Among English Language Learners: A Cognitive Linguistic Approach
}

\author{
Rod Case $^{1, *}$, Gwendolyn Williams ${ }^{2}$, Peter Cobin ${ }^{3}$ \\ ${ }^{1}$ Department of Languages, Literacy and Culture, University of Nevada, Reno, USA \\ ${ }^{2}$ Department of Curriculum and Teaching, Auburn University, Auburn, USA \\ ${ }^{3}$ Loder Elementary School, Washoe County Schools, Reno, USA
}

Email address:

rcase@unr.edu(R. Case),gmw0015@auburn.edu(G. Williams),pcobin@washoeschools.net (P. Cobin)

${ }^{*}$ Corresponding author

\section{To cite this article:}

Rod Case, Gwendolyn Williams, Peter Cobin. Problem-Solving Among English Language Learners: A Cognitive Linguistic Approach. International Journal of Elementary Education. Vol. 8, No. 1, 2019, pp. 18-25. doi: 10.11648/j.ijeedu.20190801.13

Received: December 21, 2018; Accepted: February 12, 2019; Published: May 7, 2019

\begin{abstract}
New mathematics standards ask teachers to strengthen mathematics instruction while still building upon communication skills. In today's classroom, this is complicated by the growing number of English language learners (ELLs) across the country who because they are still learning English struggle with the language of mathematics. Researchers who have addressed the question of problem-solving among ELLs have explored schema-based instruction or the use of math journals. Of these two approaches, a discussion of the ELL population is not included in research on math journals and problem solving. Within the research on schema-based instruction, research limit their findings to ELLs with math difficulties (MD). This study addresses the gap in the research on problem-solving among ELLs. A writing structure referred to as Source, Path, Goal (SPG) was used as a linguistic scaffold and type of schema-based instruction. Instruction was set in an elementary ELL classroom. Each group received a different level of scaffolded instruction: 1) instruction only treatment group, 2) instruction plus practice treatment group. Three non-parametric sign tests were conducted (one for each group) to compare preand post-test results and indicated strong support for the use of scaffolding plus practice. An analysis of the students' written explanations of how they solved their math problems only indicated an increase in problem-solving skills for instruction only treatment group, but both treatment groups increased in the depth of their mathematical thinking. Implications for practice and future research are shared.
\end{abstract}

Keywords: English as a Second Language, Problem Solving, Cognitive Linguistics

\section{Introduction}

An ongoing discussion in the teaching of problem-solving in mathematics to young children has been the question of how to teach students to deconstruct the language of the problem, parse out the most important elements and form a plan to solve it. The dominant approach within the research has been schema-based instruction (SBI). SBI emerged in response to the practice of teaching students General Solution Instruction (GSI). According to Xin, Jitendra and DeatlineBuchman [1], GSI emphasizes language comprehension and often relies on the 'key word' strategy in which students are taught to identify a key word which then can be used to guide them through the problem-solving process. Words and phrases such as 'add up to' or 'result', for instance, suggest the act of addition and thus a problem-solving plan. In addition to being unreliable indicators of such operations, e.g., 'result', for instance, could just as easily suggest multiplication as it does addition, Parmar, Cawley and Fazita [2] find that this kind of strategy does not engage students in a deeper examination of the problem-solving process. Rather, students are only focused on which mathematical operation to use-division, multiplication, addition or subtractioninstead of why they are using a particular operation.

In contrast, Xin et. al., [1] explain that research into SBI explores the use of diagrams designed to facilitate the process of problem-solving. The following principles guide SBI. The first is that instruction is given directly and focused 
on the workings of specific problem types rather than a general strategy. The most common problem types, change, compare, groups and equalizing, are used extensively in SBI research. The use of a specific type of mathematical problem allows students to represent problems visually and then create a plan for solving them. Unlike the 'key word' strategy, SBI pushes students to move beyond the practice and selection of mathematical operations and into why and how mathematical operations are used.

Second, the visual element is important. According to Jitendra, Star, Dupuis and Rodriguez [3], diagrams make the problem visible and tangible to the students and have been shown to have a positive effect on student performance. Third, instruction is given directly in how to use the particular schema. Jitendra, Harwell, Dupuis, Karl, Lein, Simonson and Slater [4] note this research and confirm that direct meta-cognitive instruction, such as monitoring and reflecting during problem solving, can improve or enhance reasoning.

Finally, meta-cognitive instruction does not have to be limited solely to the monitoring of mathematical processes. It can also include attention to the use of language in mathematics. This is particularly true for English language learners (ELLs), but there is little within SBI research which focuses on the ELL student population. Moreover, what little that is available examines ELL students with math difficulty (MD). In a recent example, Driver and Powell [5] report on the outcomes of an intervention study with nine ELLs. The intervention included direct skills instruction as well as instruction in what Driver et. al., [5] named culturally and linguistically responsive instruction' (CLRI). CLRI drew on the principles of SBI described above but, outside of SBI practices, also included a time for students to connect math problems to students' own experiences and ideas as well as to current trends in popular culture and their own heritage cultures. The results, while limited by the small sample size, demonstrated gains in problem solving skills. Research by Driver et. al., [5], however, did not include linguistic modifications to the intervention other than allowing students to use their native languages. As mentioned above, the research focused on ELLs with Mathematics Difficulty (MD).

This leaves an important gap in SBI research, as research into SBI among ELL students who do not have MD is missing. This is remarkable in light of the growing population of ELL students across the country. At present, ELLs make up about $10 \%$ of the public school population with seven states accounting for most of the population [6]. These states include Alaska, California, Colorado, Illinois, Nevada, New Mexico, and Texas. California has the highest percentage of ELLs among its public-school students, at 22.4 percent, followed by Nevada at 17.0 percent. Moreover, in 2017, according to the Nation's Report Card, just $14 \%$ of ELLs had reached the level of proficiency or above. In contrast, the overall percentage, which includes ELLs, of students achieving at the proficient level or above is $40 \%$.

This study examines the use of SBI in a third grade classroom of ELL students who do not have MD. The following elements of the SBI approach were accounted for in the design of the study: 1) focus on a specific type of problem, 2) the use of diagrams, 3) direct instruction in the use of the schema, and 4) the use of linguistic modifications designed for ELLs. A unit on two-step word problems in math was developed. Students were instructed in the use of a writing scaffold referred to as Source, Path, Goal (SPG). The questions examine the outcomes of using an SBI approach at two different levels of scaffolding: 1) instruction only treatment group, 2) instruction plus practice treatment, and 3) a treatment group which did not receive instruction in the SPG. Consistent with SBI practices, visuals were used which were specifically tailored to the problem type, two-step word problems in this case. Additionally, students were given direct instruction on how to use the SPG scaffold. Following a review of the research into SBI, an examination of students' writing samples, which took the form of math journals, is given.

\section{Literature Review}

\subsection{Word Problems}

Because ELLs are simultaneously learning English and content, word problems can present a special challenge. To that end, researchers have identified the specific vocabulary, grammatical and discourse patterns that present difficulties for students. Research into the grammatical and vocabulary difficulties students face is summarized by Schleppegrell [7]. At the grammatical level, Schleppegrell [7] identifies the following problem spots: noun phrases, being and having verbs, conjunctions with technical meanings and implicit logical relationships. Lowrie, Diezmann and Logan [8] add that more visual forms of language found in notations, graphs, tables and charts can present a challenge as well because the visuals have to be interpreted and translated into imprecise natural language.

Research into different instructional approaches to word problems is replete with intervention studies, many of which draw on SBI and focused on students with math difficulties (MD) [9-12]. A review of the SBI research follows. Van Garderen and Montague [12] explored the benefits of visual scaffolds in the mathematics classroom. Research was conducted with 66 sixth-graders. Of the 66 students, 22 were average achievers. Twenty-two were students with learning disabilities and the remaining 22 were identified as gifted. None were identified as ELL. Data was collected using the Math Processing Instrument (MPI). Following administration of the test, tallies were taken on the number of correct answers as well as the number and type of visual representations students made. Van Garderen and Montague [12] defined visual as a drawing that "encodes the visual appearance of an object or objects described in a problem" (p. 247). A schematic image, "represented the spatial relationships among the problem parts and included spatial transformations" (p. 247). Finally, interviews were conducted 
to determine the extent to which students used either schematic representations in their problems or visual. Findings demonstrated that students from the gifted group produced significantly more schematic representations of the problem while students who were identified with learning disabilities used significantly more visual representations of the problems than their peers. Also, the use of schematic representations was positively correlated with successful problem solving while visual representation was negatively correlated with successful problem solving.

Most recently, Jitendra, Harwell, Dupuis, and Karl [9] explored the use of schema-based instruction (SBI) among 806 students. The sample was a subset from among the 1,999 seventh-graders who participated in a larger study that Jitendra and her research partners had completed earlier [10, 11]. Jitendra, Harwell, Dupuis, Karl and Lein [11] conducted an intervention on the effectiveness of schema-based instruction on proportional problem-solving both immediately after the treatment (posttest) and 9 weeks after the treatment (delayed posttest). The PPS, developed in Jitendra et al's study [11], and the Treatment Acceptability Scale-Revised (TARF-R) were used as outcome measures for the treatment group to assess gains in various problem solving. The treatment involved six weeks of instruction in which the treatment group received schema-based instruction and the control received the 'business-as-usual' approach. Importantly, instructors taught the same content to both groups. Findings indicated significant gains for control group students on the PPS, scoring one third of a standard deviation higher. Significant gains were also made in the areas of promoting deep conceptual understanding as well as on the Ratio, and Proportion and Percent PPS subscales. These results were significant for the posttest but not the delayed posttest.

Other work into MD and problem-solving has confirmed the value of a schema-based instruction and a cognitive approach to problem solving demonstrating advantages in controlled studies of students with $\operatorname{MD}[12,13]$, but the approach is not without limitations. Despite the large sample sizes found in Jitendra et. al., [9, 11], ELLs were not well represented. Just $5.3 \%$ of the sample participants were ELLs in Jitendra et. al., [11] and 7.5\% in Jitendra et. al., [9]. A model drawn from a cognitive perspective is, of course, based upon the premise that use of a schema can free up working memory for the higher order thinking needed during problem solving [13]. This is consistent with the challenge ELLs face as they must simultaneously take on the challenge of mathematical problem solving and operating in a second language.

\subsection{Math Journals}

The research to date on the use of math journals in the elementary classroom is promising. First, math journals provide a means of looking into how students think and reason through problem solving but it is a task that requires explicit modeling and instruction [14, 15]. Martin and Polly [15] describe a study of how three fourth grade teachers implemented a journal writing component into the instruction of two-step word problems. After examining the responses students gave in their math journals to writing prompts about mathematics problem, Martin and Polly [15] concluded that students' writing and math skills improved when teachers integrated writing with mathematics instruction. Martin [16] argues that writing is critical to mathematics because it allows the student to concentrate and develop their word problem solving skills.

Second, there is emerging evidence that when math journals are combined with traditional math instruction, students' math skills improve. Kostos and Shin [14], as noted in Martin and Polly [15], similarly found that including writing as a part of math instruction enhanced performance in mathematical thinking. In Kostos and Shin's study [14], 16 second-graders participated in an action research study to measure the extent to which math journals could improve the expression of mathematical thinking. Data included math journals, interviews with the students and the use of teachers' reflective journals. Findings indicated a statistically significant improvement in pre- post-test performance assessment on math skills, an increase in the use of mathematical vocabulary and, finally, an increase in the amount of information provided to teachers as a result of the use of math journals.

When research into math journals and problem solving is considered as a whole, what direction should future research take? Martin and Polly [15] suggest that future research should bring problem solving and writing together. Given the level of detail of research into word problems, this seems a thoughtful direction. However, research into the workings of math journals needs the level of articulation that SBI offers in their protocols such as DISC which, because of its connection to cognitive theory, can give some important insights into how learning takes place. As such, this research is set in the elementary ELL classroom and examines the use of math journals in a unit of study on word problems. A cognitive linguistic framework is introduced within the instruction of word problems as a means of deepening the connections between the two bodies of research.

\section{Theoretical Framework}

Many math educators and researchers draw on the concept of metaphor, as described by Lakoff and Núñez [17], in many ways as a construct for analysis. According to Font, Godino, Planas and Acevedo, by using metaphor, individuals can use the properties of real-life objects to discuss mathematical concepts [18]. Wood [19], for instance, employed the concept of metaphor to distinguish the different ways that students conceptualized their growing understanding of fractions. Metaphor, according to Kalyuga [13], expresses complex or abstract ideas (the target) by linking them to a more familiar or concrete notion (the source). Wood [19] gives an example of a person 'getting into' or 'getting out' of trouble to "structure the abstract domain of trouble" (p. 17). The metaphor that is evoked here is the container. It is useful 
because it allows the speaker to describe trouble as a "bounded space" (p. 17) that one can get into or out of.

Kalyuga [13] describes many more metaphors that help elucidate the connections between language and mathematical problem solving, but we ultimately decided to use the source, path, goal (SPG) metaphor as a means of scaffolding math journal writing. The SPG scaffold describes how directed motions or movement are goal-directed and patterned. The SPG metaphor, according to Kalyuga [13], is a basic schema. As such, it functions as a building block of cognition that is developed in early childhood and used without conscious attention by the individual. All individuals, for instance, divide an activity into an internal state (source), a sequence of steps (path) and an outcome (goal). This schema develops in infancy and by the time an individual reaches grade school it is an unconscious cognitive resource that can be applied to general problem solving. When applied specifically to solving math problems, source is the information given in the problem. The path is the sequence of actions that the student carries out, and the goal is the final solution.

In math, the SPG framework provides a three-part template for journal writing that scaffolds the task of solving word problems. A two-step word problem from the pre-test is below which can be used to illustrate its application.

Elena has a collection of postcards. She arranges her postcards into 6 groups. There are 9 cards in each group, and she has 2 postcards left over. How many postcards does Elena have in her collection?

The first part, the source, asks the learners to describe what they know about the problem, sometimes referred to as the given information. In the example above, it would include the fact that there are 6 group with 9 cards in each group, and 2 are left over. Depending on the strategy the student uses, the path may consist of first multiplying $6 \times 9$ and then adding 2 , which leads to the goal of knowing that there are 56 cards total. Or the path may consist of drawing 6 circles, putting 9 tally marks inside each circle, counting the tally marks, and adding 2 more to get 56. SPG paths are not necessarily efficient.

As a structure for student responses, SPG has competition. Two other student response types are frequently used (and accepted) in the classroom. The simplest is Answer-is-aNumber, e.g. "56." In addition to being quick and easy linguistically, these responses earn prestige among peers by making the work look effortless. Only slightly more sophisticated is Answer-is-an-Operation, e.g. "I multiplied." In single-step word problems, they key to the solution is generally to select the correct operation. For simple problems, response type might be regarded as a matter of style; however, as problem complexity increases, student thinking (and responding) increasingly depends on putting actions in sequence. When students respond without using Sequence, they preclude the possibility of building a line of reasoning from Source to Path to Goal. Can we prepare students to utilize SPG?

\section{Method}

\subsection{Question and Purpose}

The purpose of the study was to develop a stronger understanding of how students' mathematical thinking might be improved with instruction, practice and the use of written scaffolds. Additionally, given the connections between literacy and mathematics established by previous research [20-22], this study also aimed to understand the extent to which using written scaffold might improve performance on word problems. To that end, the following research questions were asked.

1. Do any of the instructional treatments support the development of SPG?

2. Do any of the instructional treatments show a relationship to students' correctly solving two-step word problems?

\subsection{Participants}

The participants included 74 third grade students enrolled in the same elementary school. The school has a high enrollment of English learners, many of whom struggle with word problems as taught using the Everyday Math curriculum used in the mainstream lessons. All participants in the study were English learners, and the treatment instruction was provided during their ELL instructional time. The total instruction time was approximately 300 minutes (25 minutes a day, 4 days a week, for 3 weeks). Instruction occurred during English Language Arts time for group work, so it was a supplement to the math instruction. The lessons were designed to meet the language of mathematics standard of WIDA, the consortium for English language development of which this state is a member. Most students were below national norms in math ability on the Measures of Academic Progress (MAP) test.

Participants were divided into three groups. Students were purposefully assigned to balance English language proficiency (as measured by the overall WIDA ACCESS test, with 6 being fully proficient and 5 being the minimum for exiting EL services) and math ability (as measured by the MAP scores, with 203 being average for the end of third grade).

1. The control group ( $\mathrm{N}=25$, average overall ACCESS 4.5, average MAP math 191) received no instruction in SPG.

2. Treatment group $1(\mathrm{~N}=24$, average overall ACCESS 4.4, average MAP math 196) received explanations and models of SPG but did not practice it.

3. Treatment group $2(\mathrm{~N}=25$, average overall ACCESS 4.5, average MAP math 197) received explanations and models of SPG and a few minutes each day to practice it.

\subsection{Data Collection}

Data was collected from a number of different sources in a number of ways. To inform the formation of the three groups 
and control for levels of English language proficiency, scores from the ACCESS English proficiency scores in reading, writing, listening and speaking levels. An overall score, which is the average of all reading, writing, listening and speaking, was calculated as well. To account for differences in the levels of math proficiency, standardized scores on students' math skills were collected as well from the Smarter Balance Assessment Consortium (SBAC). The SBAC is given yearly.

First, pre- and post-tests were given to the students at the beginning and at the end of the study. The pre- and post-test provided two sources of data. The first was a measure of to what extent the students improved their scores on two-step word problems. The pre- and post-tests were each a single two-step word problem that were independent of the teacherwritten story, "The Mystery of the Green Dog", described below. The problem asked the students to solve the problem and write out their explanation. Scores on pre- and post-test were tallied and then compared across groups.

The second source of data was a measurement of the extent to which the SPG metaphor scaffolded instruction increased or did not increase students' use of sequential clauses (See below for an explanation of sequential clauses) in their writing was taken. The decision to measure changes in the use of sequential clauses as opposed to use of the SPG metaphor as a whole was based on a careful reading of Lakoff and Núñez [17]. Lakoff and Núñez [17] explain that because the SPG metaphor is a basic schema it is shared pattern of cognition as it relates to problem solving that is acquired as children develop cognitively, socially and physically. As a problem-solving metaphor, it provides a natural extension into mathematics. As such, all of the children who have reached school-age have acquired the SPG metaphor. The question is to what extent are the students employing the SPG metaphor in any given problem given different levels of scaffolding. The use of sequential clauses is an indicator of the extent to which students have drawn on the SPG metaphor, more specifically suggesting that the students have established a Source and begun detailing the Path stage of problem-solving.

Finally, the students' math journals were collected after each lesson. These were analyzed to find changes in the amount of language produced from day to day, the depth of their explanations and the accuracy of their responses. Counts of the number of steps were also taken. The overall goal was to contextualize the findings from the pre- and posttest results.

\subsection{Instructional Materials}

In this study, the researchers developed a three-chapter story about sailors and pirates. One chapter was covered each week. Each chapter was approximately 600 words long. Each week, students read one chapter and discussed three math problems that were related to the story-line. Since the objectives of the lesson fell within the domain of the Language of Mathematics standards, the bulk of the time was devoted to reading and comprehending, discussing, and explaining. The math problems were intentionally made difficult in order to motivate a meaningful discussion. The problems were not of the form used on the pre- and post-test with groups of items and a remainder, as we wanted to avoid simply teaching students a script for solving this kind of problem.

\subsection{Treatment: Metaphor-Based Instruction Across the Three Groups}

Instruction for the three treatment groups was identical for the first 20 minutes of each lesson, and the different treatments affected only the last 10 minutes (often less, depending on the pace). For the control group who received no treatment, discussion continued and time permitting, a follow-up problem was given. For treatment group 1 (exposure only) the final 10 minutes consisted of reviewing a kid-friendly version of SPG ("say what the problem is, then give each step until you reach the solution") and discussing and comparing two written explanations of a problem from a previous lesson, one with SPG sequencing and the other without. For treatment group 2 (exposure plus practice) the final 10 minutes consisted of reviewing the kid-friendly version of SPG, discussing and comparing two written explanations of a problem from a previous lesson, and having students write an explanation of the problem in this lesson. The last day of the week students in all groups worked on solving and explaining a challenge problem on their own.

\subsection{Analysis}

Two analyses were done to determine if the instructional treatments exhibited support for the development of SPG and if there was a relationship to students correctly solving twostep word problems. An analysis of the students' written explanations of how they solved their math problems was done answer the first query. Three non-parametric sign tests were conducted (one for each group) to compare pre- and post-test results. Student responses for the pre- and post-test were decomposed into clauses. Sequence is the occurrence of multiple actions that are not simultaneous, so a clause-byclause analysis is used to determine which responses made use of sequence. For example, Response (A) displays sequence (4 sequential clauses), while Responses (B) and (C) do not.

Response (A) I draw 6 groupsthen I put 9I count it all togetherIt made 54.

Response (B) 1 is left out

and it would not be equal

and she put it in 2 groups.

Response (C) You have to add it.

The second analysis of the data involved a non-parametric measure in order to determine the relationship, if any, between the number of sequential clauses produced and the correct answer. The number of clauses in each student's response was calculated. A clause had to be written in words, as opposed to numbers, and had to contain a verb. Answers that only included numbers, while valid expressions of 
understanding, were not counted as clauses. Answers that included a mix of numbers and a verb were understood as clauses.

\section{Result}

The findings revealed strong support for the use of scaffolding with practice. Three non-parametric sign tests were conducted (one for each group) to compare pre- and post-test results. The results of the current study demonstrated changes in the number of steps in explanations produced across the three groups, while treatment group 2 showed significant gains in the production of either lexical or numerical steps in their written explanations. The following section explains how the findings address the research questions posed in this study.

Do any of the instructional treatments support the development of SPG?

SPG entails much more than just sequence, but sequence is requisite for its construction. The results are analyzed at two levels. Following table 1, on the pre-test, $56 \%$ of students used sequence, and this grew to $72 \%$ post-test. The control group went down $1 \%$ over the course of the study, Treatment group 1 (exposure only) went up 17\%, and Treatment group 2 (exposure and practice) went up 32\%. Furthermore, students with a WIDA level $<5.0(\mathrm{~N}=47)$ growing from $45 \%$ to $66 \%$. Thus the findings indicated strong support for the use of scaffolding with practice as an important step in developing SPG.

Second, the findings demonstrate significant gains for treatment group 2 and supported the need for practice and direct instruction when using scaffolding. The control group showed 11 negative differences, 9 positive differences and 5 ties. The test was not significant at $p=.82$. Treatment group 1 demonstrated 9 negative differences, 7 positive differences and 8 ties with a $\mathrm{p}=.80$. Findings from treatment group 2 resulted in 5 negative difference, 15 positive differences and 6 ties with a $p=.04$. Thus, treatment group 2 was the only group in which there was a significant difference between pre- and post-test production of clauses.

Table 1. Pre-post-test Results on Two-step Word Problems.

\begin{tabular}{llll}
\hline & Control Group & Treatment Group 1 & Treatment Group 2 \\
\hline Pre-Test & $2 / 26$ & $4 / 24$ & $7 / 26$ \\
Post Test & $13 / 26$ & $12 / 24$ & $9 / 26$ \\
\hline
\end{tabular}

Do any of the instructional treatments show a relationship to students' correctly solving two-step word problems?

As can be seen in table 1, findings demonstrated gains for the pre- to post-test performance for treatment group 1 and the control group but only small gains for the treatment group 2. While this might suggest a lack of support for the use of the SPG, further analysis of the students' work over the course of the unit of study suggests otherwise. Examples from the students' written explanations of their math problems suggest that treatment group 2 demonstrated an increased depth in the explanations of or their problem solving. The examples in table 2 show the trajectory of one student from the pre-test, through one question from the unit of study to the post-test.

Table 2. Sample of Student Trajectory from Pre-to Post-test.

\begin{tabular}{l}
\hline Question \\
\hline Elena has a collection of 6 postcards. She arranges them into6 groups. There are 9 cards in each group, and she has 2 \\
postcards left over. Explain how to find the number of postcards Elena has. (Pre-Test $4 / 8$ ) \\
On the boat, Jair has an anchor that weighs 40 pounds and lots of coconuts that weigh 5 pounds each. They want to see if the \\
giant octopus weighs 100 pounds. How can Jair weigh the octopus? (Unit of study, $4 / 8$ ) \\
There 6 people on the Green Dog. Each person got 8 gold bars gold bars in each circle. I \\
$\begin{array}{ll}\text { and there were } 5 \text { gold bars left over. Explain how many gold bars I counted the } 5 \text { left over } & \text { I drew } 6 \text { circles. I put } \\
\text { were in the trunk. (Posttest, } 5 / 19) & \text { counted each gold bar. }\end{array}$ \\
\hline
\end{tabular}

The first question is taken from the pre-test. Here the question asks the students to complete a two-step problem which involves them in sorting, multiplication and finding a remainder. The student does not offer an explanation of how he solved the problem (Path), but rather provides a faulty solution that probably involved multiplying 9 and 6 together and then coming to an incorrect solution. The second problem is taken from the unit of study. The students are asked to deduce how Jair, the young hero in the story, would weigh an octopus that Jair caught with his Uncle. The problem is that the scale that they have on the boat only goes up to 60 pounds. The response is short---if not comical---and certainly not mathematical. More importantly, it suggests that the student has confused solving the problem in the story, a scale which is limited to 60 pounds, with the mathematical problem.

A month later, the student is given a similar problem. It is a two-step word problem that requires multiplication and finding a remainder. The response he gives, however, is much longer and anchored in a mathematical explanation. A clear focus on Path can be noted in a reading of the answer as the student moves step-by-step through the steps he took to solve the problem. In this case, solution was correct. While it was the case that 17 other students did not get the correct answer, the data did show an increase in the use sequential clauses and, by extension, an increased focus on detailing the problem-solving process. 


\section{Discussion}

This study explored how different levels of scaffolding influence students' performance on two-step word problems among ELLs and contributes to both bodies of research. The first finding from the current study builds upon research in Kostos and Shin [14] and Martin and Polly [15] who noted that while math journal present a worthwhile endeavor that can readily be linked to instruction in literacy, it is also a task that requires explicit modeling and instruction. Results from this study confirm and extend this finding, demonstrating the important role of scaffolding. Results from the pre- post-test measurements showed significant gains in the production of sequences for students who participated in the treatment group with practice in the number of steps they produced in their writing. Analysis of the math journal assignments demonstrated a similar pattern among the treatment plus practice group. Unlike their peers in the control group, by the end of the three weeks the treatment plus practice increased the number of sequential clauses in their math journals.

A second finding demonstrated a strong increase in the number of correct answers from for the treatment group 1 but only small gains for treatment group 2 from pre- to post-test. Analysis of the students' work throughout the unit of study as well as pre- and post-test gains, however, suggested that students gained in the length and relevance of their responses. A possible explanation for this finding is that the emphasis on including a Source, Path and Goal in a response would suggest that the students had begun to focus more of their efforts into creating a specific kind of explanation that is centered in mathematical thinking. After writing out longer and more focused explanations, students may have simply assumed that their answers were correct.

Findings from this study suggest that providing a scaffolding for problem solving such as the SPG metaphor instruction may play a part in increasing and enhancing mathematical thinking. To be sure, students can give an equation without any text and demonstrate mathematical thinking, but for the majority of the students in this study mathematical thinking was most evident when written text accompanied numerical equations. Beyond linguistic scaffolding, our study suggested that scaffolding that directed the mathematical procedures of the student were beneficial in helping the student arrive at the correct mathematical conclusion. Such a finding supports earlier research where Kostos and Shin [14] and Martin and Polly [15] used the term 'mathematical thinking' to describe student writing about problem-solving. They found that math journals and providing description of problem-solving provided opportunity for students to deepen their mathematical thinking, but they did not specify how that might be measured.

By extension, by drawing on the use of the SPG metaphor and the more specific measurement of sequences, this study contributes to the discussion of problem solving as discussed in the research on problem solving [10-12] in at least two important ways. First, current research into a schema-based and cognitive approach to problem solving has centered on developing a schema of problem types (e.g., change, combine, compare). In Jitendra et. al., [9], for instance, students were taught ratio, proportion, and percent/percent of change within the broad domain of proportion. In contrast, this study drew on cognitive linguistics and offered an examination of a problem-solving schema. While problemsolving strategies are well documented among math educators, using SPG in the math class is unique in that it is a general problem-solving strategy that emerges in early childhood. As such, it is not necessary to teach it explicitly. Within this research, the treatment only needed provide opportunities for the students to apply and practice identifying and describing the Source, Path and Goal.

Notwithstanding the value of a deeper understanding of mathematical thinking, a number of questions remain about the workings of word problems and scaffolding. First, because the participants' English language proficiency did not vary widely, this study did not report on the question of how second language proficiency may have factored into the students' ability to produce more turns. The contemporary measure of English language proficiency used by the school district in which this research took place is known as WorldClass Instructional Design Assessment (WIDA) would suggest that students of higher levels of proficiency would produce more language and, by extension more steps. The question is particularly relevant in light of of research which extols the value of literacy-based instruction and math, but sets those recommendations outside of a discussion of second language proficiency. Future research which compared the work of students at different levels of proficiency would certainly add to the research on scaffolding and word problems. Second, the question of why students did not show strong gains from pre- to post-test on the number of correct answers needs more research. Given the observation that the students gained in mathematical thinking, it seems logical that gains in finding the correct answers should follow. It is possible that the relatively short duration of the study may have played a role in this, not giving students in treatment group 2 enough time to make noticeable gains on the posttest. A direction for future research then might be to use a delayed post-test design to test this hypothesis.

Finally, despite the attention that is given to building communication skills in mathematics [23], it is clear not enough has been done to meet the needs of ELLs. The research which explores instructional approaches to word problems $[10,12]$, for instance, is centered on ELLs with MD. While detailed and important, the research on learners with math difficulties is challenging to apply to the ELL population who may have a very different set of instructional needs. Research into math journals, while potentially valuable and clearly focused on EL students, is still limited. Clearly, findings from both help to inform the question of how to close the achievement gap for ELLs in mathematics. This study has been an attempt to draw on both and, hopefully, close the achievement gap EL students face in mathematics. 


\section{Conclusion}

The study suggests at least two possible conclusions. First, the linguistic scaffolding that the SPG metaphor provided is a necessary addition to the discussion of SBI. To date, not enough SBI research has explored the potential benefits of linguistic scaffolding among ELL students who are not MD. This is surprising in light of what is known about the foundational role of linguistic scaffolding in ELL instruction. Schleppegrell [7], as noted above for instance, provides an excellent overview of challenges of using academic language and there is much by Echevarria, Vogt [23] and describing the scaffolding-based approach called Sheltered Instructional Observation Protocol (SIOP). A second conclusion is that instructional scaffolding and practice is key for SBI instruction among ELL students. Teachers who increase scaffolding and practice can reasonably expect to see evidence of deeper mathematical thinking in their students' writing as well as writing that approximates the SPG metaphor and thus problem solving.

\section{References}

[1] Xin, Y., Jitendra, A. K., \& Deatline-Buchanan, A. (2005). The effects of mathematical word problem-solving on middle school students with learning problems. The Journal of Special Education, 39 (3), 181-192. Retrieved February/March.

[2] Parmar, R. S., Cawley, J. F., \& Frazita, R. R. (1996). Word problem-solving by students with and without mild disabilities. Exceptional Children, 62, 415-429.

[3] Jitendra, A. K., Star, J. R., Dupuis, D. N., \& Rodriguez, M. C. (2013). Effectiveness of schema-based instruction for improving seventh-grade students' proportional reasoning: A randomized experiment. Journal of Research on Educational Effectiveness, 6, 114-136. http://dx.doi.org/ $10.1080 / 19345747.2012 .725804$.

[4] Jitendra, A. K., Harwell, M. R., Dupuis, D. N., Karl, S. R., Lein, A. E., Simonson, G., \& Slater, S. C. (2015). Effects of a research-based mathematics intervention to improve seventhgrade students' proportional problem solving: A cluster randomized trial. Journal of Educational Psychology, 107, 1019-1034. doi: 10.1037/edu0000039.

[5] Driver, M. K. \& Powell, S., (2017). Culturally and linguistically responsive schema intervention: Improving word problem solving for English language learners with mathematics difficulty. Learning Disability Quarterly, 40 (1) 41-45.

[6] English Learners in Public Schools (2018). The Condition of Education. National Center for Educational Statistics. Retrieved https://nces.ed.gov/programs/coe/indicator_cgf.asp.

[7] Schleppegrell, M. J. (2007). The linguistic challenges of mathematics teaching and learning: A research review. Reading \& Writing Quarterly: Overcoming Learning Difficulties, 23:2, 139-159. doi: 10.1080/10573560601158461.

[8] Lowrie, T., Diezmann, C. M. \& Logan, T. (2011). Understanding graphicacy: Students' making sense of graphics in mathematics assessment tasks. International
Journal for Mathematics Teaching and Learning, 12, 3. Retrieved from http://www.cimt.org.uk/journal/index.htm.

[9] Jitendra, A. K., Harwell, M. R., Dupuis, D. N., Karl, S. R., (2017). A randomized trial of the effects of schema-based instruction on proportional problem-solving for students with mathematics problem-solving difficulties. Journal of Learning Disabilities, 50 (3) 322-336.

[10] Jitendra, A. K., Star, J. R., Rodriguez, M., Lindell, M., \& Someki, F. (2011). Improving students' proportional thinking using schema-based instruction. Learning and Instruction, 21 (6), 731-745. doi: 10.1016/j.learninstruc.2011.04.002.

[11] Jitendra, A. K., Harwell, M. R., Dupuis, D. N., Karl, S. R., Lein, A. E., Simonson, G., \& Slater, S. C. (2015). Effects of a research-based mathematics intervention to improve seventhgrade students' proportional problem solving: A cluster randomized trial. Journal of Educational Psychology, 107, 1019-1034. doi: 10.1037/edu0000039.

[12] [Van Garderen, D., \& Montague, M. (2003). Visual-spatial representation, mathematical problem solving, and students of varying abilities. Learning Disabilities Research \& Practice, 18 (4), 246-254. doi: 10.1111/1540-5826.00079.

[13] Kalyuga, S. (2006) Assessment of learners' organised knowledge structures in adaptive learning environments. Applied Cognitive Psychology, 20(3), 311-320. doi: 10.1002/acp.1247.

[14] Kostos, K., \& Shin, E. (2010). Using math journals to enhance second graders' communication of mathematical thinking. Early Childhood Education Journal, 38 (3), 223-231. doi: 10.1007/s10643-010-0390-4.

[15] Martin, C. \& Polly, D. (2016). Examining the impact of writing and literacy connections on mathematics learning. Investigations in Mathematics Learning, 8 (3), 59-74. doi: 10.1080/24727466.2016.11790354.

[16] Martin, C. L. (2015). Writing as a tool to demonstrate mathematical understanding. School Science \& Mathematics, 115 (6), 302-313. doi: 10.1111/ssm.12131.

[17] Lakoff, G. \& Nuñez, R. E. (2000). Where mathematics come from: How the embodied mind brings mathematics into being. New York: Basic Books.

[18] Font, V., Godino, J. D., Planas, N., \& Acevedo, J. I. (2010). The object metaphor and synecdoche in mathematics classroom discourse. For the Learning of Mathematics, 30 (1), 15-19.

[19] Wood, M. B. (2010). Not understanding Andy: A metaphorical analysis students' resistance to learning. For the Learning of Mathematics, 30 (3), 17-22.

[20] Liedtke, W. W., \& Sales, J. (2001). Writing tasks that succeed. Mathematics Teaching in the Middle School, 6(6), 350-355.

[21] Moschkovich, J. N. (2015). Academic literacy in mathematics for English learners. The Journal of Mathematical Behavior, 40, 4362. doi: 10.1016/j.jmathb.2015.01.005.

[22] Ntenza, S. (2006). Investigating forms of children's writing in grade 7 mathematics classrooms. Educational Studies in Mathematics, 61 (3), 321-345.

[23] National Council of Teachers of Mathematics (Ed.) (2000). Principles and Standards for School Mathematics. National Council of Teachers of Mathematics. 